Article

\title{
Effect of Ionic Polymer Membrane with Multiwalled Carbon Nanotubes on the Mechanical Performance of Ionic Electroactive Polymer Actuators
}

\author{
Joohee Kim ${ }^{1}$, Minjeong Park ${ }^{1}$ (D), Seonpil Kim ${ }^{2}$ and Minhyon Jeon ${ }^{1, *}$ \\ 1 Department of Nanoscience and Engineering, Center for Nano Manufacturing, Inje University, \\ Gimhae 50834, Korea; 21wngml@gmail.com (J.K.); mjpark9121@gmail.com (M.P.) \\ 2 Department of Military Information Science, Gyeongju University, Gyeongju 38065, Korea; seonpil@gu.ac.kr \\ * Correspondence: mjeon@inje.ac.kr; Tel.: +82-055-320-3672
}

Received: 20 November 2019; Accepted: 7 February 2020; Published: 10 February 2020

check for updates

\begin{abstract}
Ionic electroactive polymer (IEAP) actuators have received interest because of their advantageous properties, including their large displacement, high energy density, light weight, and low power consumption under a low electric field. However, they have a low blocking force under driving, and it is difficult to control the thickness of the ionic polymer membrane. In this study, an IEAP actuator is fabricated using a Nafion membrane with added multiwalled carbon nanotubes to increase the blocking force. A heat press two-step process is also developed to produce a constant and uniform membrane. The fabricated Nafion membrane with $0.2 \mathrm{wt} \%$ multiwalled carbon nanotubes has the largest displacement and highest blocking force. As a result, the developed heat press two-step method can be used in various polymer-casting fields, and the fabricated carbon nanotube-based IEAP actuators can serve as useful references in fields such as flexible robotics and artificial muscles.
\end{abstract}

Keywords: ionic electroactive polymer; Nafion membrane; multiwalled carbon nanotubes; heat press two-step process; blocking force

\section{Introduction}

The advantageous properties of ionic electroactive polymer (IEAP) actuators include their large displacement, high energy density, light weight, and low power consumption under low electric fields [1-4]. Typically, IEAP actuators are composed of an ionic polymer membrane sandwiched between two noble metal electrodes [5,6], and the diffusion of hydrated cations within the membrane under an applied voltage and the associated electrostatic interactions induce bending.

Nafion membranes have excellent ionic conductivity and chemical and thermal stability, and they are suitable up to a thickness of $180 \mu \mathrm{m}$ for application as ionic membranes in IEAP actuators [7]. Commonly, IEAP actuators are limited by the commercially available Nafion thicknesses (e.g., N115, N117, and N1110), and thus various studies on controlling their thickness using a Nafion solution have been reported [6,8-17]. Furthermore, since the thickness of the membrane significantly affects its properties and experimental behavior, it is highly important to fabricate consistent and uniform membranes like those that are commercially available membranes in order to compare and verify experimental results. For these reasons, $N, N^{\prime}$-dimethylformamide (DMF) was used to cast the Nafion membranes in this study. DMF is highly compatible with the Nafion tetrafluoroethylene backbone, and thus enables the formation of a membrane in which the Nafion molecules do not aggregate [17]. It is also used as a solution for carbon nanotube (CNT) dispersions such as for surfactants [18-20]. However, in the Nafion membrane casting process, it proved to be difficult to acquire a uniform 
membrane with a controlled thickness. So, after the casting process, a new method of controlling the thickness through additional processes was needed. The heat press is good because it can process heat and pressure at the same time, but the fine and constant thickness control method needs to be further investigated. Therefore, the process using the metal plate and the heat press was further performed after the casting to obtain a uniform membrane having a controlled thickness.

Moreover, IEAP actuators have significant drawbacks such as low actuation bandwidth, low blocking force, and poor durability [21-24]. Their low blocking force has particularly hindered the practical applicability of IEAP actuators, necessitating studies on increasing their blocking force. The thicker membrane layer or the thicker electrode layer can cause a displacement decrease of the actuator [8-10], and the stiffer electrode layer can cause cracks on the electrode surface, and poor durability of the actuator in operation. Therefore, the application of various materials such as graphene, fullerene, carbon-metals, and graphene oxide to the actuator is studied to increase blocking force or improve actuation performances without reducing displacement [11-16,25-28]. Among various proposed additive materials, multiwalled carbon nanotubes (MWCNTs) have shown high chemical and thermal stability, excellent tensile strength and stiffness, and high conductivity and heat resistance. The excellent properties of the MWCNTs improve the mechanical, electrical, and thermal properties of various polymers [29-32]. The MWCNT was used to increase the blocking force while maintaining the displacement. However, acid treatments are commonly required in the dispersing process; these treatments damage actuators and affect their mechanical properties owing to the resulting CNT structural defects, which are undesirable for further actuator applications [33]. In contrast, Nafion can be used as an alternative to acid treatment to overcome the disadvantages arising from the chemical functionalization and physical dispersion of CNTs. Furthermore, dispersion occurs due to hydrophobic interactions between Nafion and CNTs [20], and thus MWCNTs have been considered as additives in Nafion membranes $[23,24,34]$. The resulting Nafion membranes with MWCNTs (N-MWCNT) are easily fabricated and have tunable stiffness and high ion transport. N-MWCNT also have the potential to enhance the blocking force and response speed of IEAP actuators.

Since IEAP is flexible, small, and lightweight, it is mainly developed for miniaturized and lightweight robots. Lee and Jain studied the movement of artificial muscle fingers using EMG signals, respectively $[35,36]$. As well as artificial human muscle simulation, much research has been done on robots as well as artificial human muscle simulation that simulates aquatic life such as jellyfish and fish [37-39]. Thus, the driving force is an essential factor in developing the application as an artificial muscle of a living organism.

In this study, we developed a heat press two-step (HPTS) process to obtain Nafion membranes with uniform thicknesses and fabricated N-MWCNT-based IEAP actuators. We prepared N-MWCNT membranes with a thickness of $200 \mu \mathrm{m}$ by the developed HPTS process accounting for the fact that the membrane thickness was reduced by approximately $10 \%$ when the actuators were manufactured with the paper electrodes [7]. We created N-MWCNT membranes with various MWCNT weight ratios for IEAP actuators and observed their thermal and mechanical properties.

\section{Experimental}

\subsection{Materials}

DMF was purchased from Sigma-Aldrich (St. Louis, MI, U.S.), and MWCNTs were purchased from Carbon Nanomaterial Technology Co., Ltd. (Pohang, Gyeongbok, South Korea). A Nafion solution (20 wt\%; D2021) was purchased from Dupont (St. Wilmingtoon, Delaware, U.S.), and Nafion membranes were cast using mixtures of the Nafion solution (16 g), DMF (5 wt $\%)$, and MWCNTs $(0.0 \sim 1.0 \mathrm{wt} \%)$. Graphene oxide (GO) and a silver nanowires (Ag NWs) solution were purchased from Grapheneall Co., Ltd. (Siheung-si, Gyeonggi-do, South Korea) and DUKSAN Hi-Metal (Ulsan, Gyeongnam, South Korea), respectively, and electrodes were used. Lithium chloride purchased from Sigma-Aldrich (St. Louis, MI, U.S.) and 1-ethyl-3-methylimidazolium trifluoro-methyl-sulfonate 
(ionic liquid) purchased from Merck KGaA (Darmstadt, Hesse, Germany) were used for actuator ion exchange.

\subsection{Fabrication of N-MWCNT Membranes}

The developed HPTS method employs a hot press and metal plate in two steps. Figure 1a shows step 1 , in which the approximate film thickness was determined by the weight of the dispersion. Nafion solutions containing DMF and MWCNTs were mixed using a probe sonicator, and the mixture was poured into a preheated casting mold $\left(9.0 \times 9.0 \mathrm{~cm}^{2}\right)$. The filled mold was heated at $40{ }^{\circ} \mathrm{C}$ for over $12 \mathrm{~h}$ to vaporize the volatile components of the Nafion solution (deionized water and acetone) and thus obtain the casted N-MWCNT membrane. Next, in step 2 (Figure 1b), the casted N-MWCNT membrane from step 1 and support metal plates of the desired thickness were placed between substrates with high heat resistance and strength and were heat-pressed at $140{ }^{\circ} \mathrm{C}$ and $10 \mathrm{MPa}$. Herein, the metal plate that was used was not deformed by heat and pressure, had a clean surface, and was thinner than the membrane cast in step 1. Conditions of the process such as time, temperature and pressure were optimized through repeated experiments. Finally, we obtained N-MWCNT membranes with uniform thicknesses by the HPTS method.

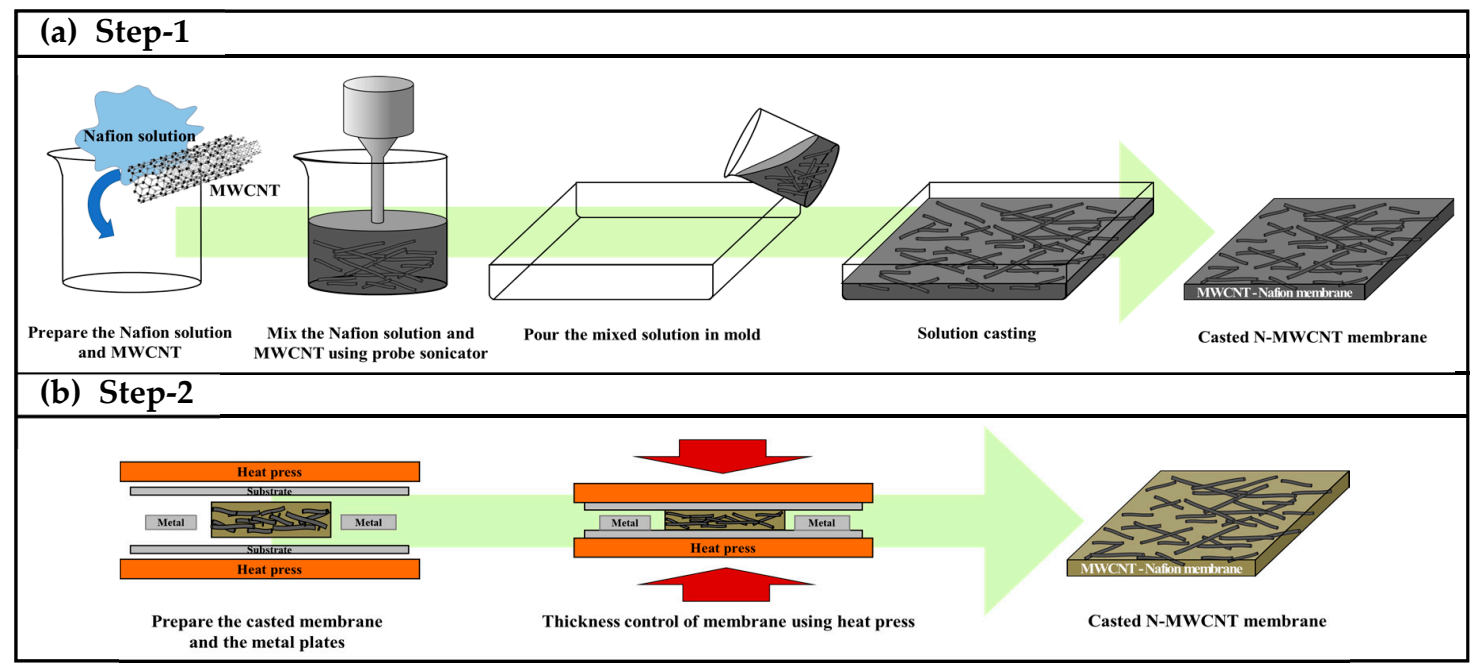

Figure 1. Schematics diagram of Nafion membranes with MWCNTs (N-MWCNT) membrane fabrication process by heat press two-step (HPTS) method (a) step-1 and (b) step-2.

\subsection{Fabrication of N-MWCNT-Based Actuators}

Actuators were manufactured using the N-MWCNT membranes and GO-Ag NWs electrodes. We used the GO-Ag NWs (weight ratio 1:2.5) paper electrodes that Yoo, S. studied [40], which were made using a vacuum filtration system. Each N-MWCNT membrane fabricated by the HPTS method was placed between the GO-Ag NWs paper electrodes and attached using the heat press to yield the N-MWCNT-membrane-based actuator. The size of fabricated actuator was $4.0 \times 0.5 \mathrm{~cm}^{2}$.

\subsection{Analytical Techniques}

We created N-MWCNT membranes with various MWCNT weight ratios for IEAP actuators and observed surface morphologies and thicknesses using field emission scanning electron microscopy (FE-SEM; S-4300, Hitachi, Tokyo, Japan) and a Micrometer analyzer. The thermal stabilities of the fabricated membranes were assessed by thermo-gravimetric analysis (TGA), and the mechanical performances of the N-MWCNT-based IEAP actuators were analyzed using a driving characteristics analyzer and tensile strength machine. The actuation performances of the actuators were measured using a laser displacement sensor (ZS-LD80, OMRON Korea, Seocho, Seoul, South Korea) and load 
cell sensor. The displacement was analyzed to the changing distance of the laser on the surface of the actuator when AC voltage was applied to the actuator. The blocking force was analyzed to the contacting load cell sensor with the surface of the actuator when DC voltage was applied to the driver.

\section{Results}

Nafion membranes for use as ionic polymer membranes in actuators were made using evaporation and HPTS methods, and were compared. As shown in Figure 2a, the surface of the Nafion membrane made by evaporation method was not flat or uniform, and it is clear that the image behind the Nafion membrane is distorted. In contrast, the surface of the Nafion membrane made by HPTS method was smooth; the image is clear, well transmitted, and not distorted (Figure 2b).
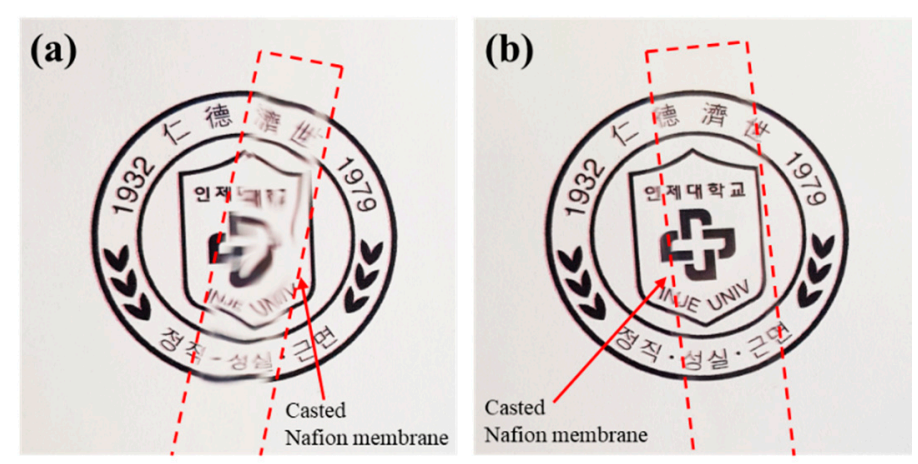

Figure 2. Photographs of Nafion membranes fabricated by (a) evaporation method and (b) HPTS method.

Figure 3 shows the SEM cross-sectional images of the Nafion membrane fabricated with $15 \mathrm{~g}$ of the Nafion solution measured at three different areas. Figure 4 a shows the average thicknesses and standard deviations of the membranes fabricated by evaporation method according the amount of Nafion solution $(10,12$, and $15 \mathrm{~g})$. The membranes fabricated by evaporation did not have uniform thicknesses and also showed large thickness variations between samples with the same weights of added Nafion. Therefore, it was not possible to obtain the desired membrane thickness. Herein, because the required Nafion membrane thickness was $200 \mu \mathrm{m}$, we manufactured the membrane using a $15 \mathrm{~g}$ Nafion solution having the sufficient thickness to which the pressing process can be applied. Figure 3a-c shows SEM cross-sectional images of the evaporation-fabricated Nafion membrane, and Figure 3d-f shows those of the HPTS-fabricated membrane. It is clear from the images that the thickness of the evaporation-fabricated membrane was nonuniform, whereas the HPTS-fabricated membrane exhibited a uniform thickness. Figure 3g-i shows SEM cross-sectional images of the HPTS Nafion membrane fabricated with MWCNTs at $1.0 \mathrm{wt} \%$, which also had a uniform thickness.

Figure $4 \mathrm{~b}, \mathrm{c}$ shows the thicknesses of the Nafion membranes fabricated using a $15 \mathrm{~g}$ Nafion solution and N-MWCNT $1.0 \mathrm{wt} \%$ by both evaporation and HPTS methods. The thickness deviations derived from nine experiments of the evaporation-fabricated Nafion and N-MWCNT $1.0 \mathrm{wt} \%$ membranes were 18.2 and 87 times larger, respectively, than those of the corresponding HPTS-fabricated membranes. Despite the addition of MWCNTs, the casted HPTS Nafion membranes had uniform thicknesses of $200 \mu \mathrm{m}$. These results show that our HPTS method is more suitable than the evaporation method for producing uniform Nafion membranes and is extensively applicable to various other efforts, such as controlling the thickness of polymers. 

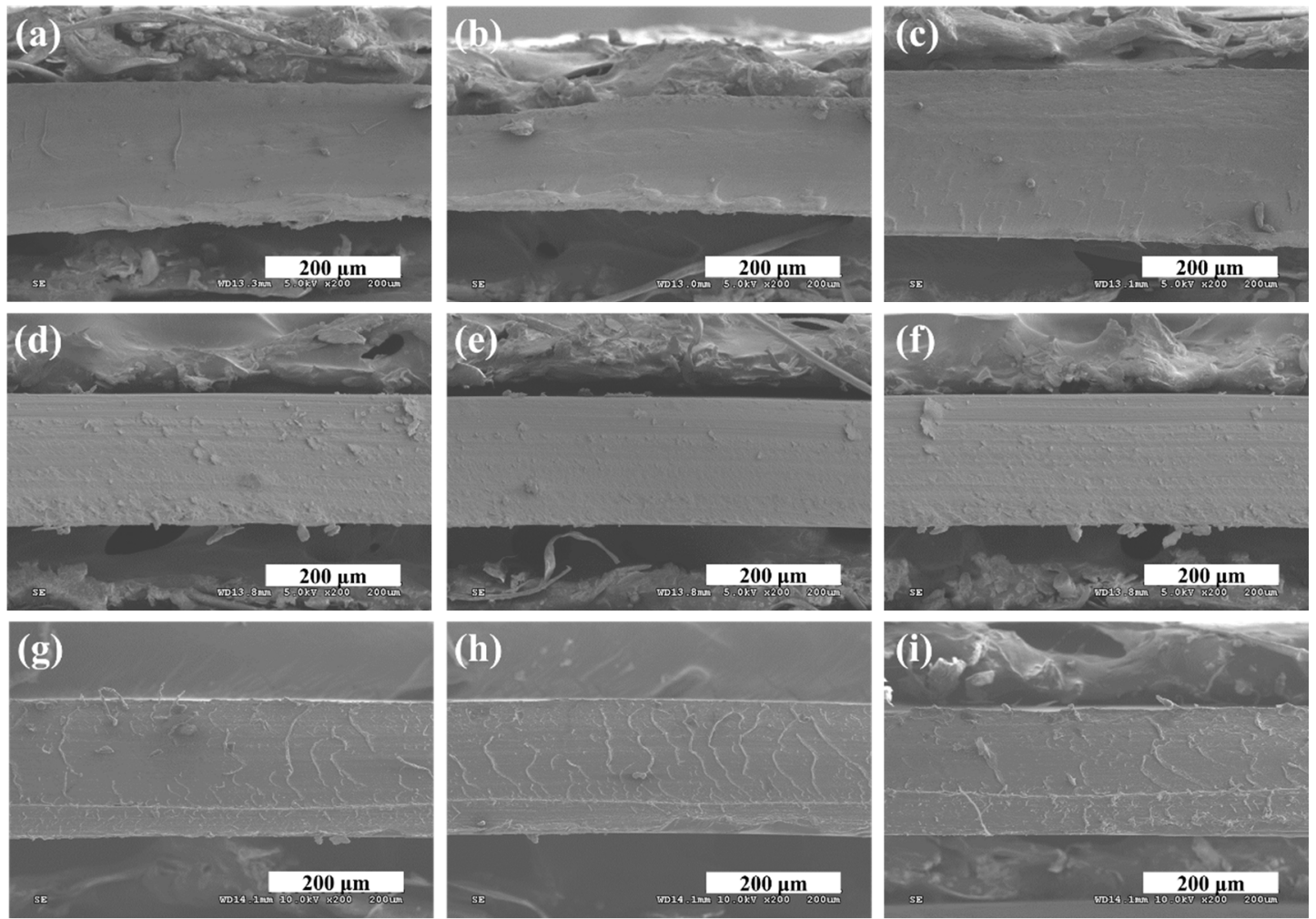

Figure 3. Cross-sectional SEM images of (a-c) evaporation-fabricated Nafion membrane without multiwalled carbon nanotubes (MWCNT), (d-f) HPTS-fabricated Nafion membrane without MWCNT, and (g-i) HPTS-fabricated Nafion and $1.0 \mathrm{wt} \%$ MWCNT composite membrane.
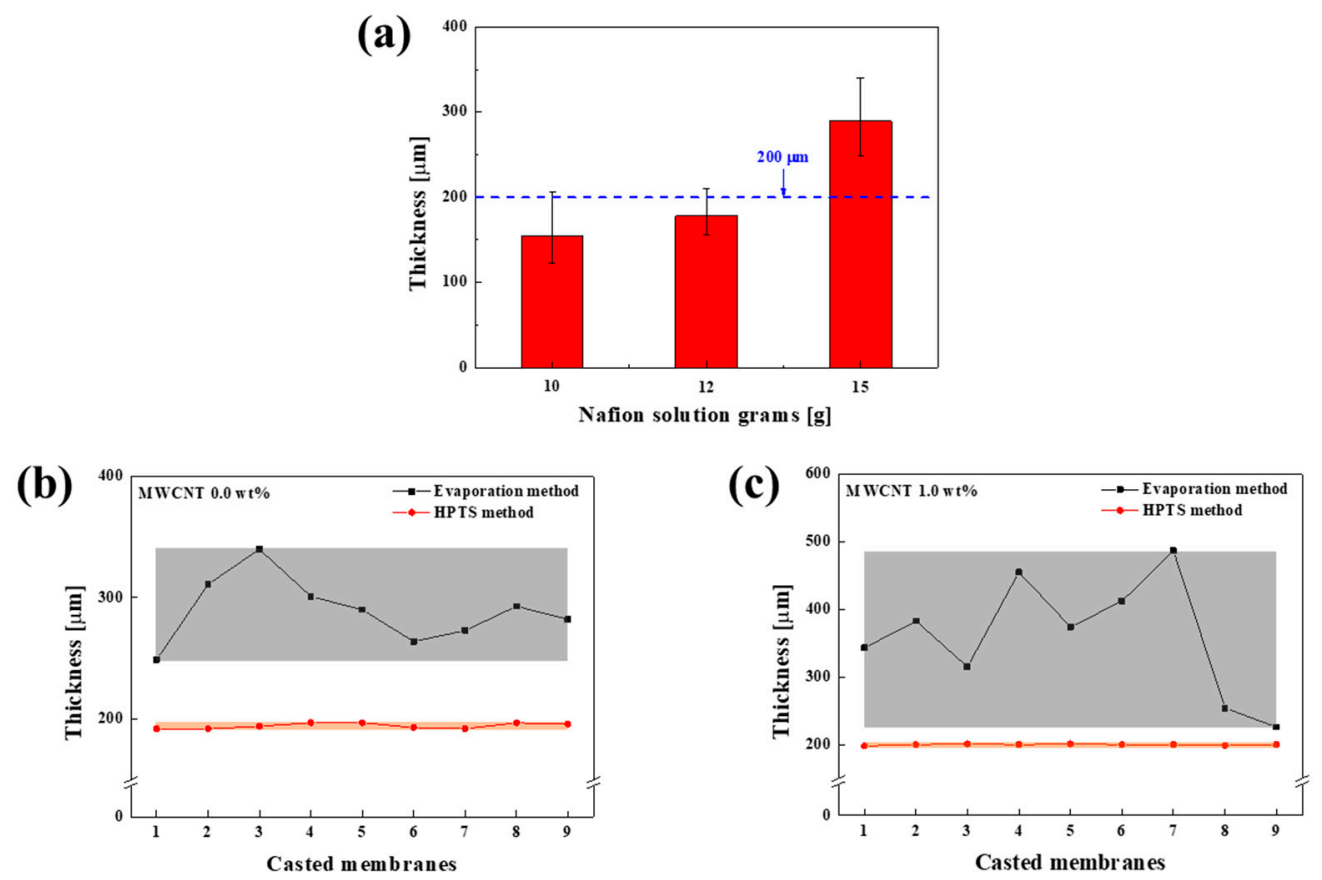

Figure 4. Thicknesses of casted Nafion membranes: (a) fabricated by evaporation method with different amounts of Nafion solution (10, 12, and $15 \mathrm{~g}$ ); fabricated by evaporation (black) and HPTS (red) (b) with a $15 \mathrm{~g}$ Nafion solution and (c) with $1.0 \mathrm{wt} \%$ MWCNTs added to a $15 \mathrm{~g}$ Nafion solution. 
Figure 5 shows cross-sectional SEM images of the HPTS N-MWCNT membranes. The N-MWCNT $1.0 \mathrm{wt} \%$ membrane had an aggregated surface (Figure 5f), and thus we fabricated N-MWCNT membranes with different MWCNT weight ratios from 0.0 to $1.0 \mathrm{wt} \%$ and compared their properties. The SEM images show that MWCNT and Nafion were evenly mixed in general, but a greater MWCNT weight ratio led to a partially aggregated surface (the white circles in the figure). The Nafion membrane made with MWCNT at $0.2 \mathrm{wt} \%$ (N-MWCNT $0.2 \mathrm{wt} \%$ ) had the smoothest surface and showed no aggregation. This result implies that $0.2 \mathrm{wt} \%$ MWCNTs is the most suitable amount for achieving a good dispersion in the Nafion solution.
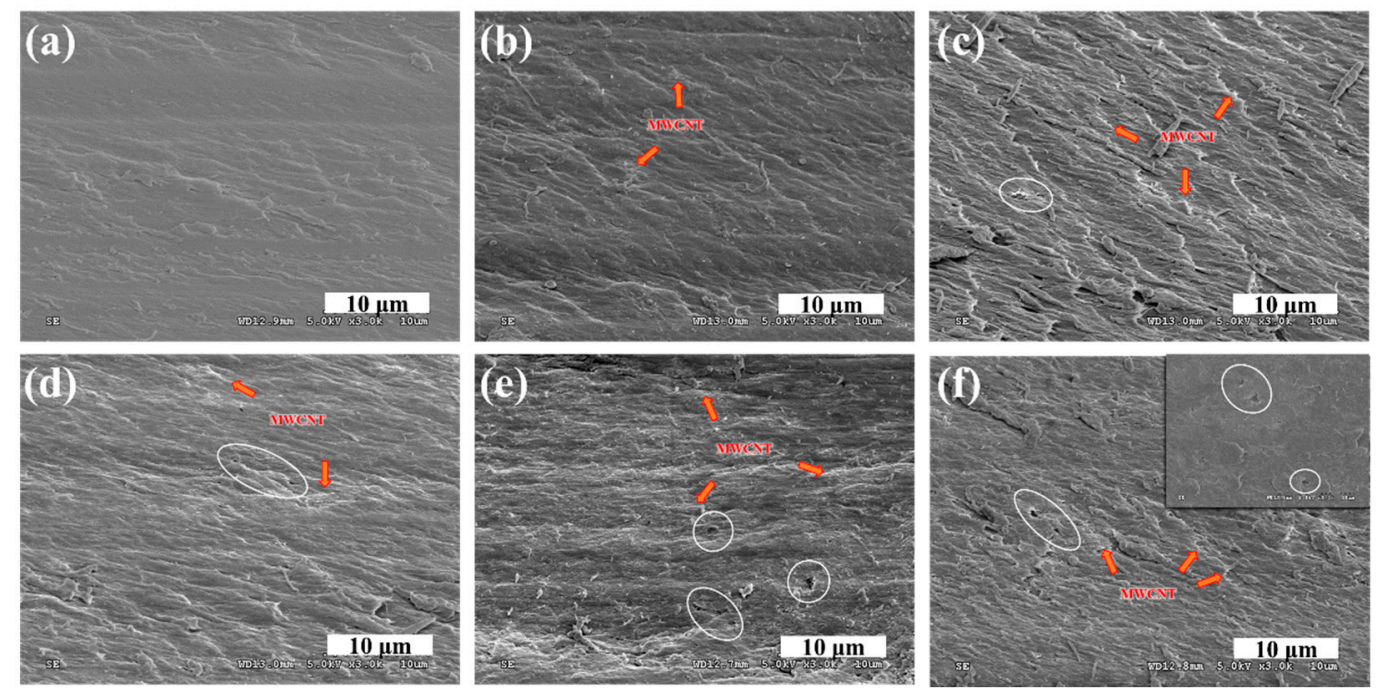

Figure 5. Cross-sectional SEM images of N-MWCNT membranes with different MWCNT weight ratios: (a) $0.0 \mathrm{wt} \%$, (b) $0.2 \mathrm{wt} \%$, (c) $0.4 \mathrm{wt} \%$, (d) $0.6 \mathrm{wt} \%$, (e) $0.8 \mathrm{wt} \%$, and (f) $1.0 \mathrm{wt} \%$.

The tensile strengths of the Nafion and N-MWCNT membranes (Figure 6a) were also analyzed. The tensile strength of the N-MWCNT $0.2 \mathrm{wt} \%$ membrane was $22.3 \mathrm{MPa}$, which was 1.19 times higher than that of the Nafion membrane without MWCNTs. The N-MWCNT $0.2 \mathrm{wt} \% \mathrm{membrane}$ also had the highest tensile strength of all the N-MWCNT membranes, owing to its nonaggregated surface.
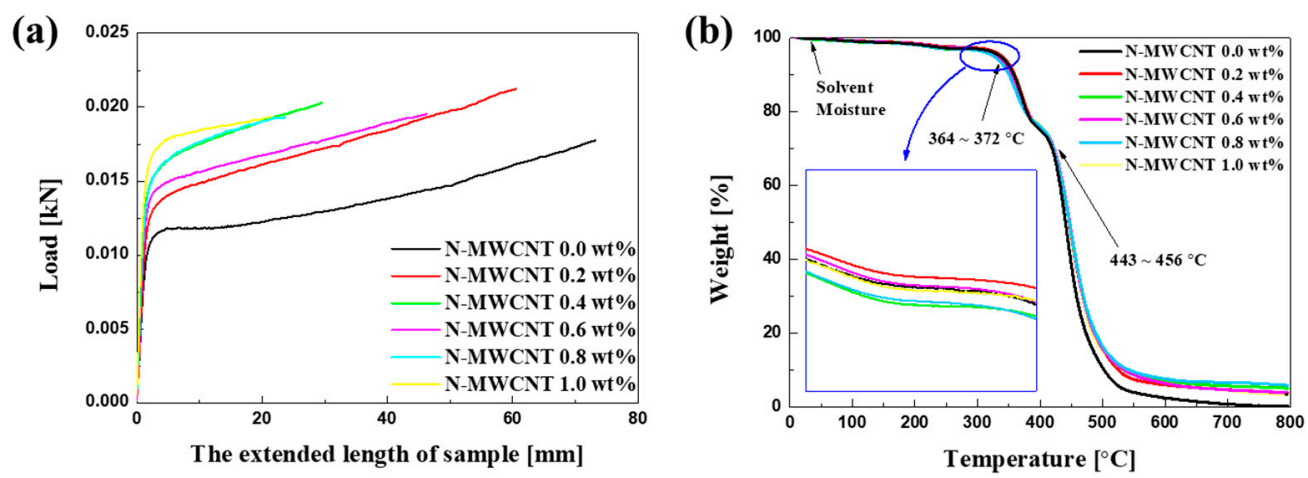

Figure 6. (a) Tensile moduli and (b) thermo-gravimetric analysis (TGA) curves of casted HPTS Nafion membrane without MWCNTs and N-MWCNT membranes with different MWCNT weight ratios (inset: enlargement of $364-372{ }^{\circ} \mathrm{C}$ TGA range).

The obtained TGA results indicate the thermal stability of the Nafion and N-MWCNT membranes (Figure 6b). Weight loss occurred in all the Nafion membranes between approximately 370 and $450{ }^{\circ} \mathrm{C}$. The initial weight loss above $300{ }^{\circ} \mathrm{C}$ could be due to the decomposition of sulfonic acid groups, and the remaining majority of the weight loss is due to decomposition of carbon-fluorine bonds [41]. The N-MWCNT $0.2 \mathrm{wt} \%$ membrane had a higher thermal stability than both the Nafion membrane 
without MWCNTs and the other N-MWCNT membranes (inset of Figure $6 b$ ). These results show that the thermal stability of the N-MWCNT $0.2 \mathrm{wt} \%$ membrane was improved because the MWCNTs were well dispersed in the Nafion membrane.

The GO-Ag NWs paper electrode that we used had an electrical conductivity $(\sigma)$ of about $9615 \mathrm{~S} / \mathrm{cm}$, a sheet resistance of about $250 \mathrm{~m} \Omega / \mathrm{sq}$., and a thickness of about $14 \mu \mathrm{m}$. The actuator was fabricated with the N-MWCNT membrane. Figure 7 shows the actuation performances of the Nafion-based actuator without MWCNTs and the N-MWCNT-based actuators. The N-MWCNT $0.2 \mathrm{wt} \%$-based actuator featured a displacement of $0.845 \mathrm{~mm}$, which was 2.34 times larger than that of the Nafion-based actuator without MWCNTs (Figure 7a). We also observed that the maximum displacement decreased as the MWCNT weight ratio increased. Figure $7 \mathrm{~b}$ shows the actuation performances of the N-MWCNT $0.2 \mathrm{wt} \%$-based actuator under various input voltages $\left(1\right.$ and $\left.2 \mathrm{~V}_{\mathrm{AC}}\right)$ at $0.2 \mathrm{~Hz}$. The displacement of the $\mathrm{N}$-MWCNT $0.2 \mathrm{wt} \%$-based actuator decreased but still stably actuated under $1 \mathrm{~V}_{\mathrm{AC}}$. Figure $7 \mathrm{c}$ shows the bending curvatures and blocking forces of the casted Nafion-based actuator and N-MWCNT-based actuators with different MWCNT weight ratios under $2 \mathrm{~V}_{\mathrm{DC}}$. The bending curvatures were calculated using Equation (1):

$$
\kappa=1 / R=2 \delta /\left(1^{\wedge} 2+\delta^{\wedge} 2\right)
$$

where $R, \delta$, and $l$ are the radius of curvature, tip displacement, and actuator free length, respectively. The free length in the actuation measurement is the length from the position of the electrode in contact for voltage application to the laser point measuring the displacement with actuator surface standard. The N-MWCNT $0.2 \mathrm{wt} \%$-based actuator had the highest blocking force of $0.086 \mathrm{mN}$, which was 1.6 times larger than that of the Nafion-based actuator without MWCNTs (Figure 7c). These results show that a high actuator performance resulted from the addition of MWCNTs, yielding a Nafion membrane with high strength and thermal stability. Herein, we observed that the N-MWCNT $0.2 \mathrm{wt} \%$-based actuator had the best actuation performance compared with the Nafion-based actuator and other N-MWCNT-based actuators.
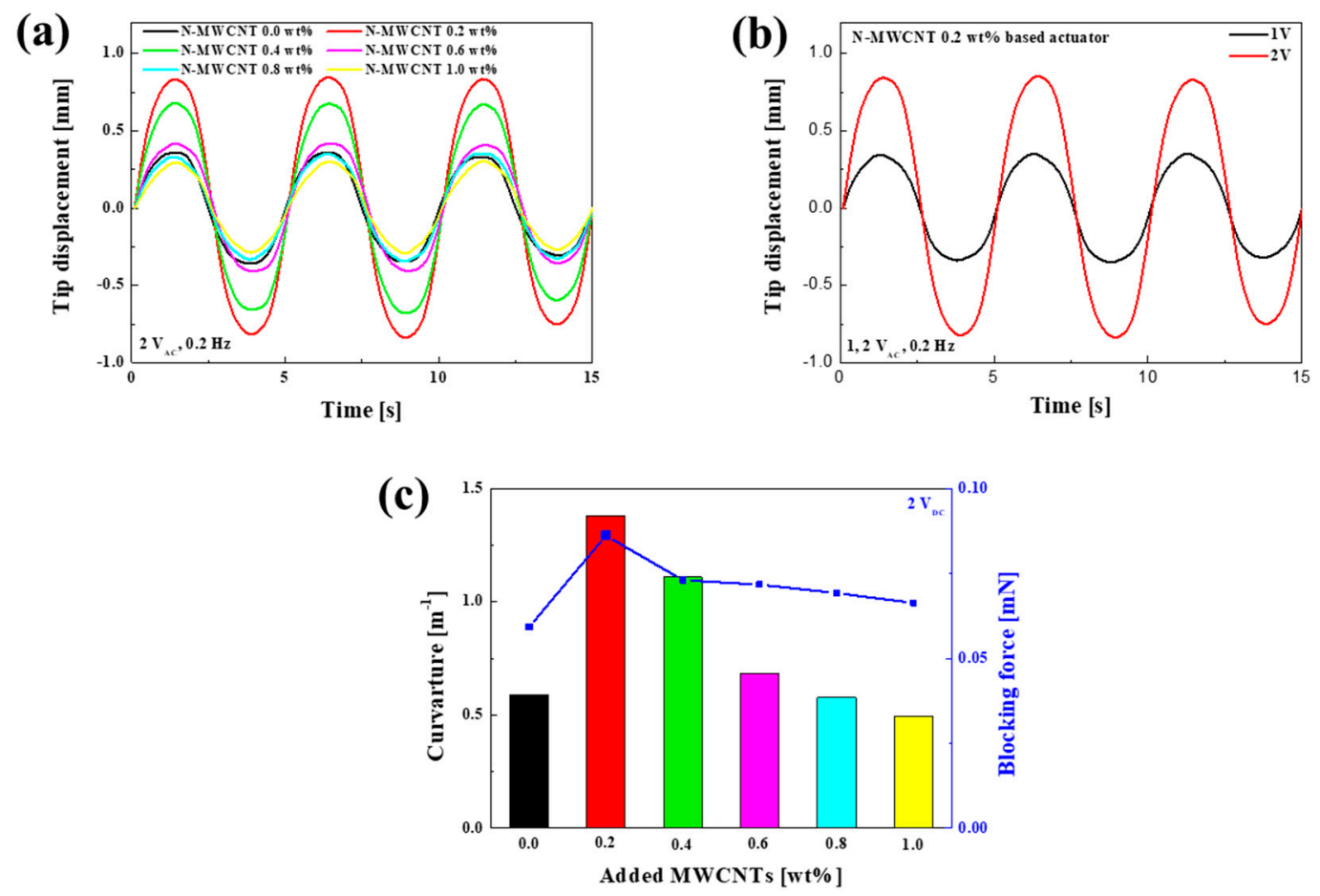

Figure 7. (a) Tip displacements of casted Nafion-based actuator and N-MWCNT-based actuators with different MWCNT weight ratios under $2 \mathrm{~V}_{\mathrm{AC}}$ and $0.2 \mathrm{~Hz},(\mathbf{b})$ tip displacements $\left(1\right.$ and $\left.2 \mathrm{~V}_{\mathrm{AC}}, 0.2 \mathrm{~Hz}\right)$ of $\mathrm{N}$-MWCNT $0.2 \mathrm{wt} \%$-based actuator, and (c) curvatures and blocking forces of casted Nafion-based actuator and N-MWCNT-based actuators with different MWCNT weight ratios under $2 \mathrm{~V}_{\mathrm{DC}}$. 


\section{Conclusions}

In this study, we developed an HPTS method to produce Nafion membranes with consistent and uniform thicknesses, and we fabricated N-MWCNT membranes using different MWCNT weight ratios to increase the actuator blocking force. The deviations in thickness of the HPTS-fabricated Nafion and N-MWCNT membranes were 18.2 and 87 times lower, respectively, than those of the corresponding membranes fabricated by evaporation method. Therefore, the Nafion and N-MWCNT membranes fabricated by the HPTS method had highly uniform thicknesses and surfaces. We determined the suitable MWCNT weight ratio to be approximately $0.2 \mathrm{wt} \%$ for application to IEAP actuators. The N-MWCNT $0.2 \mathrm{wt} \%$-based actuator had a tensile strength of approximately $22.3 \mathrm{MPa}$, which was 1.19 times higher than that of the Nafion-based actuator without MWCNTs and was the largest among the tensile strengths of all the N-MWCNT-based actuators tested. It also had the highest thermal stability among all the actuators, both with and without MWCNT addition. In addition, the blocking force and displacement of the N-MWCNT $0.2 \mathrm{wt} \%$-based actuator were $0.086 \mathrm{mN}$ and $0.0845 \mathrm{~mm}$, which are 1.6 and 2.34 times higher, respectively, than those of the actuator without MWCNTs. As a result, the addition of MWCNTs afforded the actuator with good mechanical and chemical properties, including high strength and thermal stability of the Nafion membrane. However, if the MWCNT weight ratio exceeds the suitable amount, sufficient dispersion is not achieved, which can decrease the membrane mechanical and chemical properties. Finally, the developed HPTS method can effectively be used in research on polymer materials that requires controlled thicknesses, and the $\mathrm{N}$-MWCNT $0.2 \mathrm{wt} \%$-based actuator can be applied to flexible devices, artificial robots, and flexible actuator technologies.

Author Contributions: Conceptualization and data curation, J.K and M.P.; formal analysis, J.K., M.P. and S.K.; resources, S.K. and M.J.; writing—original draft preparation, J.K. and M.P.; writing—review and editing, J.K., M.P. and M.J.; supervision, M.J. All authors have read and agreed to the published version of the manuscript.

Funding: This research was supported by a grant to Bio-Mimetic Robot Research Center Funded by Defense Acquisition Program Administration, and by Agency for Defense Development (UD190018ID).

Conflicts of Interest: The authors declare no conflict of interest.

\section{References}

1. Zaghib, K.; Charest, P.; Guerfi, A.; Shim, J.; Perrier, M.; Striebel, K. LiFePO4 safe Li-ion polymer batteries for clean environment. J. Power Sources 2005, 146, 380-385. [CrossRef]

2. Shahinpoor, M.; Bar-Cohen, Y.; Simpson, J.O.; Smith, J. Ionic polymer-metal composites (IPMCs) as biomimetic sensors, actuators and artificial muscles-a review. Smart Mater. Struct. 1998, 7, R15. [CrossRef]

3. Jung, K.; Nam, J.; Choi, H. Investigations on actuation characteristics of IPMC artificial muscle actuator. Sens. Actuators A Phys. 2003, 107, 183-192. [CrossRef]

4. Chung, C.K.; Fung, P.K.; Hong, Y.Z.; Ju, M.S.; Lin, C.C.K.; Wu, T.C. A novel fabrication of ionic polymer-metal composites (IPMC) actuator with silver nano-powders. Sens. Actuators B Chem. 2006, 117, 367-375. [CrossRef]

5. Punning, A.; Kruusmaa, M.; Aabloo, A. Surface resistance experiments with IPMC sensors and actuators. Sens. Actuators A Phys. 2007, 133, 200-209. [CrossRef]

6. Kim, B.; Kim, D.H.; Jung, J.; Park, J.O. A biomimetic undulatory tadpole robot using ionic polymer-metal composite actuators. Smart Mater. Struct. 2005, 14, 1579. [CrossRef]

7. Park, M.; Kim, J.; Song, H.; Kim, S.; Jeon, M. Effects of Thickness and MultiWalled Carbon Nanotube Incorporation on the Performance of Nafion Membrane-Based Ionic Polymer-Metal Composite Actuators. Nanosci. Nanotechnol. Lett. 2018, 10, 1121-1125. [CrossRef]

8. Kim, B.; Kim, B.M.; Ryu, J.; Oh, I.H.; Lee, S.K.; Cha, S.E.; Pak, J. Analysis of mechanical characteristics of the ionic polymer metal composite (IPMC) actuator using cast ion-exchange film. In Proceedings of the Smart Structures and Materials 2003: Electroactive Polymer Actuators and Devices (EAPAD), San Diego, CA, USA, 2-6 March 2003; Volume 5051, pp. 486-496.

9. He, Q.; Yu, M.; Song, L.; Ding, H.; Zhang, X.; Dai, Z. Experimental study and model analysis of the performance of IPMC membranes with various thickness. J. Bionic Eng. 2011, 8, 77-85. [CrossRef] 
10. Lee, S.J.; Han, M.J.; Kim, S.J.; Jho, J.Y.; Lee, H.Y.; Kim, Y.H. A new fabrication method for IPMC actuators and application to artificial fingers. Smart Mater. Struct. 2006, 15, 1217-1224. [CrossRef]

11. Dai, Z.; Ansaloni, L.; Ryan, J.J.; Spontak, R.J.; Deng, L. Nafion/IL hybrid membranes with tuned nanostructure for enhanced CO 2 separation: Effects of ionic liquid and water vapor. Green Chem. 2018, 20, 1391-1404. [CrossRef]

12. Amiinu, I.S.; Li, W.; Wang, G.; Tu, Z.; Tang, H.; Pan, M.; Zhang, H. Toward anhydrous proton conductivity based on imidazole functionalized mesoporous silica/nafion composite membranes. Electrochim. Acta 2015, 160, 185-194. [CrossRef]

13. Jung, J.H.; Jeon, J.H.; Sridhar, V.; Oh, I.K. Electro-active graphene-Nafion actuators. Carbon 2011, 49, 1279-1289. [CrossRef]

14. Jung, J.H.; Vadahanambi, S.; Oh, I.K. Electro-active nano-composite actuator based on fullerene-reinforced Nafion. Compos. Sci. Technol. 2010, 70, 584-592. [CrossRef]

15. Oh, I.K.; Jung, J.H.; Jeon, J.H.; Vadahanambi, S. Electro-chemo-mechanical characteristics of fullerene-reinforced ionic polymer-metal composite transducers. Smart Mater. Struct. 2010, 19, 075009. [CrossRef]

16. Lee, S.H.; Jung, J.H.; Oh, I.K. 3D Networked Graphene-Ferromagnetic Hybrids for Fast Shape Memory Polymers with Enhanced Mechanical Stiffness and Thermal Conductivity. Small 2014, 10, 3880-3886. [CrossRef] [PubMed]

17. Lin, H.L.; Yu, T.L.; Huang, C.H.; Lin, T.L. Morphology study of Nafion membranes prepared by solutions casting. J. Polym. Sci. Pt. B Polym. Phys. 2005, 43, 3044-3057. [CrossRef]

18. Caneba, G.T.; Dutta, C.; Agrawal, V.; Rao, M. Novel ultrasonic dispersion of carbon nanotubes. J. Miner. Mater. Charact. Eng. 2010, 9, 165. [CrossRef]

19. Nguyen, T.T.; Nguyen, S.U.; Phuong, D.T.; Nguyen, D.C.; Mai, A.T. Dispersion of denatured carbon nanotubes by using a dimethylformamide solution. Adv. Nat. Sci. Nanosci. Nanotechnol. 2011, 2, 035015. [CrossRef]

20. Barsan, M.M.; Ghica, M.E.; Brett, C.M. Electrochemical sensors and biosensors based on redox polymer/carbon nanotube modified electrodes: A review. Anal. Chim. Acta 2015, 881, 1-23. [CrossRef]

21. Kim, D.; Kim, K.J.; Palmre, V. Electro-chemical operation of ionic polymer-metal composites. Sens. Actuators $B$ Chem. 2011, 155, 106-113. [CrossRef]

22. Chen, Z.; Shen, Y.; Xi, N.; Tan, X. Integrated sensing for ionic polymer-metal composite actuators using PVDF thin films. Smart Mater. Struct. 2007, 16, S262. [CrossRef]

23. Lee, J.W.; Yoo, Y.T. Preparation and performance of IPMC actuators with electrospun Nafion ${ }^{\circledR}-M W N T$ composite electrodes. Sens. Actuators B Chem. 2011, 159, 103-111. [CrossRef]

24. Levitsky, I.A.; Kanelos, P.; Euler, W.B. Electromechanical actuation of composite material from carbon nanotubes and ionomeric polymer. J. Chem. Phys. 2004, 121, 1058-1065. [CrossRef] [PubMed]

25. Kim, K.; Palmre, V.; Jeon, J.-H.; Oh, I.-K. IPMCs as EAPs: Materials. In Electromechanically Active Polymers: A Concise Reference; Springer: Cham, Switzerland, 2016; pp. 151-170.

26. Bauer, F.; Willert-Porada, M. Microstructural characterization of Zr-phosphate-Nafion ${ }^{\circledR}$ membranes for direct methanol fuel cell (DMFC) applications. J. Membr. Sci. 2004, 233, 141-149. [CrossRef]

27. Li, J.; Vadahanambi, S.; Kee, C.D.; Oh, I.K. Electrospun fullerenol-cellulose biocompatible actuators. Biomacromolecules 2011, 12, 2048-2054. [CrossRef]

28. Kim, H.J.; Randriamahazaka, H.; Oh, I.K. Highly conductive, capacitive, flexible and soft electrodes based on a 3D graphene-nanotube-palladium hybrid and conducting polymer. Small 2014, 10, 5023-5029. [CrossRef]

29. Spitalsky, Z.; Tasis, D.; Papagelis, K.; Galiotis, C. Carbon nanotube-polymer composites: Chemistry, processing, mechanical and electrical properties. Prog. Polym. Sci. 2010, 35, 357-401. [CrossRef]

30. Thostenson, E.T.; Ren, Z.; Chou, T.W. Advances in the science and technology of carbon nanotubes and their composites: A review. Compos. Sci. Technol. 2001, 61, 1899-1912. [CrossRef]

31. Moniruzzaman, M.; Winey, K.I. Polymer nanocomposites containing carbon nanotubes. Macromolecules 2006, 39, 5194-5205. [CrossRef]

32. Gong, X.; Liu, J.; Baskaran, S.; Voise, R.D.; Young, J.S. Surfactant-assisted processing of carbon nanotube/polymer composites. Chem. Mat. 2000, 12, 1049-1052. [CrossRef]

33. He, Q.; Yu, M.; Yu, D.; Ding, Y.; Dai, Z. Significantly enhanced actuation performance of IPMC by surfactant-assisted processable MWCNT/Nafion composite. J. Bionic Eng. 2013, 10, 359-367. [CrossRef] 
34. Kashiwagi, T.; Grulke, E.; Hilding, J.; Harris, R.; Awad, W.; Douglas, J. Thermal degradation and flammability properties of poly (propylene)/carbon nanotube composites. Macromol. Rapid Commun. 2002, 23, 761-765. [CrossRef]

35. Jain, R.K.; Datta, S.; Majumder, S. Design and control of an IPMC artificial muscle finger for micro gripper using EMG signal. Mechatronics 2013, 23, 381-394. [CrossRef]

36. Lee, M.J.; Jung, S.H.; Kim, G.S.; Moon, I.; Lee, S.; Mun, M.S. Actuation of the artificial muscle based on ionic polymer metal composite by electromyography (EMG) signals. J. Intell. Mater. Syst. Struct. 2007, 18, 165-170. [CrossRef]

37. Aureli, M.; Kopman, V.; Porfiri, M. Free-location of underwater vehicles actuated by ionic polymer metal composites. IEEE ASME Trans. Mechatron. 2010, 15, 603-614. [CrossRef]

38. Chen, Z.; Shatara, S.; Tan, X. Modeling of biomimetic robotic fish propelled by an ionic polymer-metal composite caudal fin. IEEE ASME Trans. Mechatron. 2010, 15, 448-459. [CrossRef]

39. Yeom, S.W.; Oh, I.K. A biomimetic jellyfish robot based on ionic polymer metal composite actuators. Smart Mater. Struct. 2009, 18, 085002. [CrossRef]

40. Yoo, S.; Park, M.; Kim, S.; Chung, P.S.; Jeon, M. Graphene Oxide-Silver Nanowires Paper Electrodes with Poly (3,4-ethylenedioxythiophene)-Poly(styrenesulfonate) to Enhance the Driving Properties of Ionic Electroactive Polymer Actuators. Nanosci. Nanotechnol. Lett. 2018, 10, 1107-1112. [CrossRef]

41. Peng, K.J.; Lai, J.Y.; Liu, Y.L. Preparation of poly (styrenesulfonic acid) grafted Nafion with a Nafion-initiated atom transfer radical polymerization for proton exchange membranes. RSC Adv. 2017, 7, 37255-37260. [CrossRef]

(C) 2020 by the authors. Licensee MDPI, Basel, Switzerland. This article is an open access article distributed under the terms and conditions of the Creative Commons Attribution (CC BY) license (http://creativecommons.org/licenses/by/4.0/). 